David Dupin

is Head of Planning at $141 \mathrm{Com}$
Keywords: Set theory, response testing, selection sampling

David Dupin

Head of Planning,

$141 \mathrm{COM}$,

121-141 Westbourne Terrace,

London W2 6JR

Tel: +44 (0)20 74790321

E-mail:

ddupin@141communications.com

\section{Direct response multi-list market testing}

David Dupin

Received (in revised form): 2 May 2000

\begin{abstract}
Marketing methodology that is not set based suggests that the marketer needs to do two things when market testing:

- ensure that the sample is an unbiased selection of the universe from which it is selected;

- ensure that the sample is big enough to produce a statistically reliable result.
\end{abstract}

Set theory suggests that these two steps are inadequate. It predicts that the test response will not be a proper estimate of roll-out responses. Bar exceptional circumstances, roll-out responses will almost always be lower.

Set theory further suggests that different methodologies need to be used depending on whether the marketing test is for direct mail, when it is possible to communicate on a net names basis, or a media format such as loose inserts, when this is not possible. Methodologies not using set theory do not differentiate between the two cases.

To summarise the approach, the marketer makes a series of test selections from external databases or lists for direct marketing test purposes. Response from each test selection is measured. This response is used as an estimate for the response that will be achieved when mailing (rolling out) to the balance of each list.

There are two possible types of datasets. In the simple case the marketer makes selections from a single database. In this instance a sample is taken from a known and finite number of records. In the complex case the marketer is making selections from more than one database, or any other media source.

The simple case is dealt with in the author's paper 'Ordering database selections by probability of response' in the Journal of Database Marketing, Vol. 7, No. $3^{1}$. This paper deals with cases when the marketer is multi-list testing.

\section{Using set theory to predict response rates when multi-list testing}

\section{Overview on set theory approach to multi-list testing}

Set theory suggests that multi-list direct marketers following the two steps outlined in the abstract will test biased samples. Roll-out responses are therefore very likely to be different and also lower - unless the marketer takes remedial action. 
The cause of the sampling bias for both direct mail and loose insert users is that individuals who are more likely to respond to the advertising are over-sampled in the test campaign. These responsive names are defined as those that appear on more than one media source.

Loose insert users are likely to suffer response depression since in the test campaign they over-sample the whole market.

\section{Duplicate names and expected response rate}

When a media source is selected, for example direct mail, it is because it is believed that the group of names on the list is more likely to respond than the universe of names available to mail.

Several criteria might be used to select such prospects. For example, a financial services company may select names from one list on the basis that they represent a close match to the psycho-demographic profile of current customers. Names may be selected from another list on the basis that they have enquired about other services and so are mail responsive in respect of financial services, or on the basis that they are subscribers to a thematic magazine title.

Let us say that a name appears on one or more of such lists. Is it likely that this name is more likely to respond positively? The answer is obviously yes, because the individual would have conformed to all or many of the targeting criteria.

Response analysis on the basis of sets has confirmed this fact. The evidence for the product tested indicates that a name which appears on two lists is twice as likely to respond as a name which appears on one list only, with triple duplicates performing three times as well. Obviously these ratios are likely to vary for each product or service marketed.

The example media used is direct mail. A simple three-list scenario is set up (Table 1). The direct marketer has tested 12,000 names each from three lists. The names selected are genuine unbiased samples from the total list. All three tests deliver a response rate of 2 per cent. Without using set theory the marketer therefore expects a 2 per cent response from the 108,000 names on each list that remain unmailed.

Table 1: The test scenario

\begin{tabular}{|llll|}
\hline & List A & List B & List C \\
\hline Test cell volume & 12,000 & 12,000 & 12,000 \\
Test response rate & $2 \%$ & $2 \%$ & $2 \%$ \\
Total list size & 120,000 & 120,000 & 120,000 \\
\hline
\end{tabular}

In Table 2, the direct marketer has identified three prospect lists. Each totals 120,000 names. List A has 50,000 names unique to that list. It shares 30,000 names in common with list $\mathrm{B}, 30,000$ in common with list $C$ and 10,000 names in common with both lists $B$ and $C$. The same principle and counts apply to lists B and C.

The inter-list duplication shown in Table 2 means that the net names universe is smaller than the gross count in Table 1. As shown in Table 3 , names that are on only one list comprise 60 per cent of the mailing universe. Names that are on two lists comprise 36 per cent of the 


\section{Actual inter-list duplication}

Actual duplication of names in sample
Table 2: Table 1 mailing universe expanded to show duplicate names

\begin{tabular}{|c|c|c|c|}
\hline & List $A$ & List B & List $\mathrm{C}$ \\
\hline List $\mathrm{A}$ & 50,000 & & \\
\hline List B & & 50,000 & \\
\hline List C & & & 50,000 \\
\hline List $A$ and $B$ & 30,000 & 30,000 & \\
\hline List $A$ and $C$ & 30,000 & & 30,000 \\
\hline List B and C & & 30,000 & 30,000 \\
\hline Lists $\mathrm{A}, \mathrm{B}$ and $\mathrm{C}$ & 10,000 & 10,000 & 10,000 \\
\hline Totals & 120,000 & 120,000 & 120,000 \\
\hline
\end{tabular}

Table 3: Mailing universe — net names by set source

\begin{tabular}{|lrr|}
\hline & & $\%$ \\
\hline List A names only & 50,000 & 20 \\
List B names only & 50,000 & 20 \\
List C names only & 50,000 & 20 \\
List A and B names & 30,000 & 12 \\
List A and C names & 30,000 & 12 \\
List B and C names & 30,000 & 12 \\
List A, B and C names & 10,000 & 4 \\
Total & 250,000 & 100 \\
\hline
\end{tabular}

mailing universe. Names that are on three lists comprise 4 per cent of the mailing universe.

From Table 1 a test mailing is conducted using a 10 per cent sample of 12,000 names per list. This would deliver the matrix of names by set source shown in Table 4.

Table 4: Test mailing by set source - gross names

\begin{tabular}{|c|c|c|c|}
\hline & List $\mathrm{A}$ & List B & List $\mathrm{C}$ \\
\hline List $\mathrm{A}$ & 5,000 & & \\
\hline List B & & 5,000 & \\
\hline List C & & & 5,000 \\
\hline Lists $\mathrm{A}$ and $\mathrm{B}$ & 3,000 & 3,000 & \\
\hline Lists $\mathrm{A}$ and $\mathrm{C}$ & 3,000 & & 3,000 \\
\hline Lists $B$ and $C$ & & 3,000 & 3,000 \\
\hline Lists $A, B$ and $C$ & 1,000 & 1,000 & 1,000 \\
\hline Totals & 12,000 & 12,000 & 12,000 \\
\hline
\end{tabular}

Every cell in the matrix is simply 10 per cent of the size of each cell in Table 2 . This would be so if a genuinely random sample were selected.

\section{Test mailing by set source as shown by the test de-duplication report}

Table 4 shows the test mailing by set source by the gross names selected. What information is available to the marketer on the nature of the universe and the sample at this stage?

The marketer will know that each list comprises 120,000 names and that the 10 per cent sample of each list is therefore 12,000. The level of inter-list duplication will be indicated by the de-duplication report run on the test sample. 
Table 4 shows the population of names in the test sample, broken down by the actual duplicate status of each name. Table 5 shows how this duplicate status is reported on by the sample de-duplication report.

\section{Reported sample duplication}

\section{How does this happen?}

Table 5: Test mailing by set source as indicated by the de-duplication report

\begin{tabular}{|lrr|} 
& Total names & \% total \\
\hline List A names only & 11,210 & 32.20 \\
List B names only & 11,210 & 32.20 \\
List C names only & 11,210 & 32.20 \\
List A and B names & 390 & 1.12 \\
List A and C names & 390 & 1.12 \\
List B and C names & 390 & 1.12 \\
List A, B and C names & 10 & .03 \\
Total & 34,810 & 100.00 \\
\hline
\end{tabular}

Table 5 shows a very significant shift from the universe (Table 3 ) in the breakdown by set (or duplicate) count. Names that appear on one list only are reported on as comprising 96.6 per cent of the test mailing universe. Names that appear on two lists seem to comprise 3.37 per cent of the test mailing universe. Names that appear on three lists seem to comprise 0.03 per cent of the test mailing universe.

At first these data do not support the summary's proposition. Table 5 analysis appears to show that duplicates are significantly under-represented in the test mailing by comparison with Table 3 . This is the exact opposite information from that required to support the theory.

\section{Test mailing by set source - Actual}

From the above, a standard de-duplication report inverts the set (duplication) reality. How does this happen?

Table 3 in conjunction with Table 5 demonstrates that duplicate counts based on test samples are valueless when used to estimate duplicate counts for the universe. However, the counts are systematically valueless, and what is really going on can be calculated from them. To do this, we need to understand how the de-duplication process works when applied to sample data.

\section{Unduplicated names}

The names that are not duplicated will be accurately counted as shown in Table 4. The de-duplication report will show 5,000 names for each list for these names.

\section{Double duplicates}

The random selection process will select 3,000 names from list A that are duplicates with list B. If all 30,000 A and B list names were picked from list B for duplicate matching, all the 3,000 list A names would be identified as duplicates. However, as a 10 per cent sample only is taken from list $\mathrm{B}$, there is only a 10 per cent chance that the names will match with the list A duplicates. The duplicate report will therefore only show $300 \mathrm{~A}$ and $\mathrm{B}$ duplicates. This is correct for the sample as there are only 300 duplicates in the samples. 
As a measure of the net names by set source shown in Table 3, the duplicate report fails to give the true picture: 2,700 of the A names will not be identified as double duplicates and the same number of $B$ names. These 2,700 names will not be considered duplicates and will be added to the 5,000 count of unduplicated names. The same process applies to $\mathrm{A}$ and $\mathrm{C}$ duplicates and $\mathrm{B}$ and $\mathrm{C}$ duplicates.

\section{Triple duplicates}

The reporting of treble duplicates from the list universes is more complex. There are 1,000 of each of these on each sample list. As this represents a 10 per cent sample, the number that will be successfully identified by de-duplication of the sample names is $1,000 \times 0.10^{2}$, which works out at just ten names. This is what the test de-duplication report will show (see Table 5).

A significantly higher proportion of treble duplicates from the list universe will be misidentified as double duplicates when the deduplication takes place on the test sample. Taking the test samples of lists $\mathrm{A}$ and $\mathrm{B}$ as an example, the probability that the A treble duplicates will match the $\mathrm{B}$ treble duplicates is $1,000 \times 0.10$, or 100 names. From the paragraph above, ten of these 100 names will be identified as triple duplicates. The remaining 90 are identified as double duplicates. The process will occur for the other possible double duplicate permutations, leading to 90 of the test sample treble duplicates being wrongly reported as $\mathrm{A}$ and $\mathrm{C}$ duplicates, and 90 wrongly reported as $\mathrm{B}$ and $\mathrm{C}$ duplicates.

The balance of the 1,000 treble duplicates will not be identified as duplicates at all. For each list, there will be 810 names reported in this way.

\section{Summary: Test mailing by set source - Actual}

From the exposition above, the 11,210 names that appear to be unique to list A on the sample de-duplication report are the total of:

- 5,000 names that are genuinely unique;

- 2,700 that are A and B duplicates that were not successfully recognised, together with the same number of $\mathrm{A}$ and $\mathrm{C}$ duplicates;

- 810 names that are duplicates with both lists $\mathrm{B}$ and $\mathrm{C}$ that were not successfully recognised.

The same count and principle apply to lists B and C.

The 390 names that appear to be A and B double duplicates are the total of:

- 300 of the 3,000 A and B duplicates that were successfully recognised;

- 90 of the A, B and C duplicates that were misidentified as A and B duplicates only.

The same counts and principle apply to $\mathrm{B}$ and $\mathrm{C}$ and $\mathrm{A}$ and $\mathrm{C}$ duplicates.

From the exposition above, the true reality by duplicate count for the test mailing can be calculated (Table 6). 
The reality of the sample ...

\section{$\ldots$ and the universe}

Table 6: Test mailing by set source - actual

\begin{tabular}{|lcr|}
\hline & Total names & $\%$ total \\
\hline List A names only & 5,000 & 14.36 \\
List B names only & 5,000 & 14.36 \\
List C names only & 5,000 & 14.36 \\
List A and B names & 5,700 & 16.37 \\
List A and C names & 5,700 & 16.37 \\
List B and C names & 5,700 & 16.37 \\
List A, B and C names & 2,710 & 7.79 \\
Total & 34,810 & 100.00 \\
\hline
\end{tabular}

The total for Table $6(34,810)$ is exactly the same as the total for Table 5 but compiled as sets.

The recompilation shows that responsive duplicates in the test mailing are significantly over-sampled. The information from the sample deduplication report is completely misleading if it is used to draw inferences about the nature of the mailing universe, as it inverts the true set structure of the test mailing. Double duplicates comprise 49.1 per cent of the test mailing universe, and treble duplicates 7.8 per cent. The comparative figures for the universe (table 2) are 36 per cent and 4 per cent respectively.

As testing has shown, duplicated names will respond much better and therefore lead to a test response rate than is higher than would be achieved for the universe as a whole.

To complete the picture, we need to break down into sets the universe of names available to us after the test mailing has been conducted (Table 7). As the test mailing will inevitably — and unavoidably - over-sample duplicate names it follows that these will be under-represented in the universe of names that remain to be mailed the roll-out universe.

Table 7: Roll-out mailing universe

\begin{tabular}{|lrr|}
\hline & Total names & $\%$ total \\
\hline List A names only & 45,000 & 20.91 \\
List B names only & 45,000 & 20.91 \\
List C names only & 45,000 & 20.91 \\
List A and B names & 24,300 & 11.29 \\
List A and C names & 24,300 & 11.29 \\
List B and C names & 24,300 & 11.29 \\
List A, B and C names & 7,290 & 3.39 \\
Total & 215,190 & 100.00 \\
\hline
\end{tabular}

Table 7 shows that names which appear on one list only comprise 62.7 per cent of the mailing universe. Names that appear on two lists comprise 33.9 per cent of the mailing universe, and names that appear on three lists comprise 3.4 per cent of the mailing universe. The difference between the percentage total breakdown of the test universe and the residual marketing universe is even bigger. This is attributable to duplicate over-sampling at test mailing stage, and will exacerbate response depression. 


\section{The consequent extent of response depression in the direct mail example}

\section{Set-based response prediction when multi-list testing}

\section{Response prediction using a non-set-based approach}

Say the test mailing for the three lists above was conducted in line with conventional methodology. Responses from multiple duplicates would not be reported separately; they would be simply be reported and analysed as coming from list $\mathrm{A}$, list $\mathrm{B}$ and list $\mathrm{C}$.

Say that the response from lists $\mathrm{A}, \mathrm{B}$ and $\mathrm{C}$ is 2 per cent and that the marketer decides to roll out to the whole of the universe with all three of the lists tested.

There are 215,190 names left to mail (see Table 7). The test response rate is 2 per cent, so the marketer will expect 4,304 responses.

\section{Response prediction using a set-based approach}

Additional assumptions are required to produce a quantified response depression example (Table 8).

Table 8: Duplicate response uplift assumptions

\begin{tabular}{|lc|}
\hline & Response uplift \\
\hline Record presence in two sets (ie on two lists) & $50 \%$ \\
Record presence in three sets (ie on three lists) & $100 \%$ \\
\hline
\end{tabular}

Response gains twice as high as this have been observed in the past. Analysis was based on coding response by duplicate observation. It indicated that record presence in two sets (lists) was likely to double response, and record presence in three or more sets would triple it.

\section{Response prediction using set theory}

In practice, test responses are boosted by the disproportionate level of responsive duplicates. This ratio will be much lower at roll out.

Adjusting for this bias to reflect the set breakdown of the residual mailing universe indicates the marketer will actually achieve a response rate of 1.81 per cent. This represents a 9 per cent drop from the test level and provides conclusive evidence that multi-list testing creates biased samples.

It must therefore be unsound to use the response rates achieved at test stage for roll-out projections.

\section{Using set theory to predict response rates when multi-list testing with loose inserts}

Exactly the same principle outlined for direct mail applies when loose inserts are tested in printed media. However, set theory produces a further counterintuitive prediction with this media type; response depression with loose inserts is likely to be significantly higher. This is because of an additional 'duplicate wastage' effect. The data used for direct mail can be used to demonstrate this.

Say that all the response information that was used for direct mail applies to a loose insert test. The direct mailing used 36,000 gross names at test stage. This came down to 34,810 names after de-duplication. 
The response rate was 2 per cent, so 695 orders were generated. There is no opportunity for a de-duplication routine when loose inserts are used. These orders would be generated by 36,000 loose inserts so the response rate is 1.93 per cent. The 'duplicate wastage' of the test will be 34,810 / 36,000 or 3.3 per cent.

At roll-out the marketer using loose insert media will use 324,000 loose inserts (360,000 media universe minus 36,000 test universe). However, Table 7 shows that only 215,190 names will remain that have not seen the advertising. The 'duplicate wastage' at roll out will therefore be $215,190 / 324,000$ or 33.6 per cent. This is a far higher proportion than would be experienced during the test phase.

From the direct mail example we had a response prediction of 3,901 sales at roll out, based on the test response level. Applying this to our insert scenario delivers a response rate of 1.20 per cent.

The overall response depression level with loose inserts is therefore 38 per cent; 9 per cent of this would be attributable to over-sampling the more responsive names at test stage (as the direct mail example), and 29 per cent to duplicate wastage.

\section{Set theory applied to multi-list testing: Summary of predictions}

\section{List selection}

The better the list selection the greater will be the response depression. Direct marketing media selection is all about targeting mailings to key prospects. The better is this list selection and targeting, the greater will be the level of duplication between list prospects.

\section{Sample sizes}

\section{Summary}

The smaller the test mailing as a proportion of the total marketing universe, the bigger will be response depression. If the test mailing is a small proportion of the marketing universe, the residual (or roll-out) universe will not be much different from the marketing universe as a whole. However, the over-sampling of names at test mailing stage will be proportional (in a complex way) to the ratio between the number of names in the test and the number of names in the universe. This will more than compensate for the relatively small duplicate count difference between the marketing universe and the residual universe.

\section{Number of lists tested}

The more lists tested simultaneously, the bigger will be the response depression. The analysis in this paper shows that the sampling bias and reporting for the triple duplicates were more extreme than for the double duplicates. The more lists tested, the greater this effect will be.

\section{Media formats}

Response depression when testing with loose inserts is likely to be significantly higher than when testing with direct mail. The reasons for this are shown above. 'Duplicate wastage' creates an additional cause for response depression, but the root cause is the same. 


\section{Set theory: General summary}

The relevance of set theory as a practical working tool for direct marketers can be summed up in Table 8. If a record is on more than one list, it fits several of the selection criteria. This is almost certain to make it more responsive, and independent analysis has confirmed this. The solution approach is based on the fact that a genuinely random test selection will produce a biased sample. The sample bias is towards the more responsive names.

\section{Reference}

1. An abstract of this can be found in Vol. 2 No. 1 of this Journal, p. 67.

\section{Appendix: Equations and terminology}

Say two lists are tested. These are termed list A and list B.

Samples from the lists are defined as a and $\mathrm{b}$.

The sizes of the lists are termed $\mathrm{N}_{\mathrm{A}}$ and $\mathrm{N}_{\mathrm{B}}$.

The sizes of the samples are termed $\mathrm{N}_{\mathrm{a}}$ and $\mathrm{N}_{\mathrm{b}}$.

The overlap (or duplication) of names on the whole lists is defined as $\mathrm{N}_{\mathrm{AB}}$.

Technical Appendix The overlap (or duplication) of the samples is defined as $\mathrm{N}_{\mathrm{ab}}$.

The overlap between names on (for example) the list A sample and the whole of list $\mathrm{B}$ is defined as $\mathrm{N}_{\mathrm{aB}}$.

Let $\mathrm{n}_{\mathrm{AB}}=$ the number in the combined sample which are on both lists. Defining the number in the combined sample which are on both lists $\mathrm{n}^{*}{ }_{\mathrm{AB}}=\mathrm{n}_{\mathrm{aB}}+\mathrm{n}_{\mathrm{Ab}}-\mathrm{n}_{\mathrm{ab}}$

The estimate for the number of names on the list A sample which are on list $\mathrm{B}$ is

$\mathrm{E}\left(\mathrm{n}_{\mathrm{aB}}\right)=\frac{\mathrm{n}_{\mathrm{a}}}{\mathrm{N}_{\mathrm{a}}}+\mathrm{N}_{\mathrm{AB}}$

Defining the estimate for the number of names on the list A sample that are on the list B sample

$\mathrm{E}\left(\mathrm{n}_{\mathrm{ab}}\right)=\frac{\mathrm{n}_{\mathrm{a}}}{\mathrm{N}_{\mathrm{A}}} * \frac{\mathrm{n}_{\mathrm{b}}}{\mathrm{N}_{\mathrm{B}}} * \mathrm{~N}_{\mathrm{AB}}=\frac{\mathrm{n}_{\mathrm{a}} \mathrm{E}\left(\mathrm{n}_{\mathrm{ab}}\right)}{\mathrm{N}_{\mathrm{A}}}$

Defining the estimate for the number of names in the combined sample which are on both lists

$$
\mathrm{E}\left(\mathrm{n}_{\mathrm{AB}}^{*}\right)=\left(\frac{\mathrm{n}_{\mathrm{a}}}{\left(\mathrm{N}_{\mathrm{A}}\right.}+\frac{\mathrm{n}_{\mathrm{b}}}{\mathrm{N}_{\mathrm{B}}}-\frac{\left.\mathrm{n}_{\mathrm{a}} \mathrm{n}_{\mathrm{b}}\right)}{\left.\mathrm{N}_{\mathrm{A}} \mathrm{N}_{\mathrm{B}}\right)} * \mathrm{~N}_{\mathrm{AB}}\right.
$$

For a three-list example, an estimate for the number in the combined sample which are on all three lists would be

$\mathrm{E}\left(\mathrm{n}^{*}{ }_{\mathrm{ABC}}\right)=\left(\frac{\left(\mathrm{n}_{\mathrm{a}}\right.}{\left(\mathrm{N}_{\mathrm{b}}\right.}+\frac{\mathrm{n}_{\mathrm{c}}}{\mathrm{N}_{\mathrm{B}}}+\frac{\mathrm{n}_{\mathrm{a}} \mathrm{n}_{\mathrm{b}}}{\mathrm{N}_{\mathrm{C}}}-\frac{\mathrm{n}_{\mathrm{a}} \mathrm{n}_{\mathrm{c}}}{\mathrm{N}_{\mathrm{A}} \mathrm{N}_{\mathrm{B}}}-\frac{\mathrm{n}_{\mathrm{b}} \mathrm{n}_{\mathrm{c}}}{\mathrm{N}_{\mathrm{A}} \mathrm{N}_{\mathrm{C}}}+\frac{\mathrm{n}_{\mathrm{a}} \mathrm{n}_{\mathrm{b}} \mathrm{n}_{\mathrm{c}} \mathrm{N}_{\mathrm{C}}}{\mathrm{N}_{\mathrm{A}} \mathrm{N}_{\mathrm{B}} \mathrm{N}_{\mathrm{C}}}\right) * \mathrm{~N}_{\mathrm{ABC}}$

From the above, the algebraic complexity that defines the duplicate estimates expands exponentially as the number of lists sampled expands arithmetically. 